\title{
Osteosíntesis en una fractura por estrés del pubis. Reporte de un caso y revisión de la bibliografía
}

\author{
Miguel Carabajal Mattar, Francisco Schumacher \\ Departamento de Ortopedia y Traumatología, Sanatorio Allende, Córdoba, Argentina
}

\begin{abstract}
RESUMEN
La fractura por estrés del pubis es un cuadro poco frecuente, se ha descrito en corredores de larga distancia y reclutas militares. Se han publicado 193 casos que fueron tratados de forma conservadora. El objetivo de este artículo es presentar un caso resuelto de manera alternativa, analizar los casos ya publicados, las enfermedades asociadas, el diagnóstico y el tratamiento. Se presenta el caso de una paciente añosa, deportista, con fractura por estrés del pubis, que no respondió al tratamiento conservador y fue tratada con cirugía. La paciente evolucionó favorablemente, retornó a sus actividades y el dolor desapareció. La indicación del tratamiento no solo debe limitarse al grado de inestabilidad de la fractura, sino que también se debe considerar el contexto médico y social del enfermo.
\end{abstract}

Palabras clave: Fractura por estrés; pubis; osteosíntesis.

Nivel de Evidencia: IV

\section{Osteosynthesis in a Pubic Stress Fracture. Case Report and Literature Review}

\section{ABSTRACT}

Pubic stress fracture (PSF) is a rare condition, described in long-distance runners and military recruits. One hundred and ninetythree conservatively managed cases have been documented in the literature. The purpose of this paper is to report an alternatively resolved case, and to review previously reported cases, associated pathologies, diagnosis, and treatment. We report a case of an elderly patient, sportingly active, with PSF, who did not respond to conservative treatment and was surgically treated. Follow-up of the patient was favorable with remission of pain and return to activities. The indication for treatment should not be limited only to the degree of instability of the fracture, but should also take into account the medical and social context of the patient.

Keywords: Stress fracture; pubis; osteosynthesis.

Level of Evidence: IV

\section{INTRODUCCIÓN}

Las fracturas por estrés son un cuadro conocido en traumatología del deporte y son más frecuentes en corredores y adultos jóvenes. La mayoría se localiza en el miembro inferior, y la tibia, el tercio distal del peroné y los metatarsianos son las localizaciones más comunes. ${ }^{1}$ La fractura por estrés del pubis es poco frecuente, se observa en corredores de larga distancia y reclutas militares, representa alrededor del $4 \%$ de todas las fracturas por estrés y es considerada de bajo riesgo. ${ }^{1-3}$

Las fracturas por estrés son el resultado final de la fatiga o la insuficiencia del hueso afectado. ${ }^{4}$ Las fracturas por fatiga ocurren luego de una sobrecarga fisiológica, cíclica y repetitiva que conduce a la aparición de microtraumas en un tejido óseo normal que carece del tiempo y la capacidad suficientes para someterse a la remodelación ósea, adaptarse a una nueva condición y reparar su corrosión. Mientras que las fracturas por insuficiencia se producen luego de una carga de baja intensidad sobre un hueso sometido a una afección sistémica que comprometa su arquitectura (corticoides, tabaquismo, radioterapia, inmovilización prolongada, hiperparatiroidismo). ${ }^{4-7}$

Recibido el 1-10-2020. Aceptado luego de la evaluación el 4-12-2020 • Dr. MIGUEL CARABAJAL MATTAR • miguelcarabajalmattar@gmail.com https://orcid.org/0000-0002-0301-0867 Cómo citar este artículo: Carabajal Mattar M, Schumacher F. Osteosíntesis en una fractura por estrés del pubis. Reporte de un caso y revisión de la bibliografía. Rev Asoc Argent Ortop Traumatol 2021;86(4):537-544. https://doi.org/10.15417/issn. 1852-7434.2021.86.4.1214 
Existen numerosos factores que contribuyen al desarrollo de esta entidad. Los factores extrínsecos se relacionan con el régimen deportivo, el tipo de deporte, los hábitos nutricionales y el tipo de terreno. ${ }^{1,8,9}$ Entre los factores intrínsecos podemos mencionar estados hormonales, baja densidad mineral ósea, masa corporal baja, aptitud física inadecuada al inicio de la actividad deportiva, variaciones anatómicas, afecciones musculares, sexo, etnia y edad. ${ }^{1,10,11}$ La fractura por estrés del pubis se presenta clínicamente como un dolor inguinal de alta sensibilidad y edema en la zona afectada luego de la práctica deportiva abrupta o repetitiva con intervalos de descanso insuficientes. $^{4}$

Existen pocas series publicadas que documenten esta lesión (Tabla) y siempre el problema se resolvió mediante tratamiento conservador. Presentamos un caso de una paciente añosa que no respondió a este manejo y fue operada, lo que permitió resolver los síntomas. Analizamos las enfermedades asociadas, el diagnóstico y el tratamiento de los casos publicados.

\section{CASO CLÍNICO}

Mujer de 72 años, exfumadora, con antecedentes de glaucoma, hipertensión arterial controlada, osteoporosis diagnosticada mediante densitometría mineral ósea y un índice de masa corporal de 21. Consulta en el Departamento de Ortopedia y Traumatología de nuestra institución por dolor inguinal derecho de 12 meses de evolución, de comienzo insidioso, sin antecedente de trauma directo. Además, refiere que practica tenis y golf semanalmente.

En el examen físico, se detecta dolor a la rotación interna y externa de la cadera derecha, dolor a la palpación del pubis derecho y dolor a la aducción contrarresistencia del miembro inferior derecho. Este cuadro inicialmente fue diagnosticado como una tendinitis insercional de los músculos aductores. Se solicitó una radiografía de pelvis (Figura 1A), en la que se observó un área radiopaca en la zona de la sínfisis púbica. Por el antecedente deportivo, la densidad mineral ósea baja y el tiempo de evolución del síntoma, se solicitó una resonancia magnética (Figura 1B y C) donde se confirmó una fractura por estrés del pubis derecho. El tratamiento inicial consistió en agentes analgésicos y antinflamatorios, sumados a reposo de actividades deportivas y rehabilitación física. Se derivó a la paciente al Servicio de Clínica Médica de nuestra institución para tener un asesoramiento del estado clínico y detectar algún factor de riesgo que pudiera influir en el tratamiento. Luego de seis meses de rehabilitación física, la paciente volvió a la consulta con limitación al caminar y un dolor de las mismas características, por lo que se decidió repetir los estudios por imágenes. Nuevamente, se observó el aumento de la radiopacidad en las radiografías simples y un idéntico foco de intensidad aumentada con edema óseo en el pubis derecho, en la resonancia magnética (Figura 2).

Luego de discutir el diagnóstico y las opciones terapéuticas con la paciente, se decidió realizar una osteosíntesis de la sínfisis del pubis con una placa de reconstrucción de huesos planos de acero inoxidable de 3,5 mm (Stryker® Trauma) y seis tornillos corticales autorroscantes de 3,5 mm (Figura 3). Se logró una fijación estable y no hubo complicaciones técnicas para su aplicación. La paciente comenzó con terapia de rehabilitación a los 15 días de la cirugía, y evolucionó favorablemente, con mejoría progresiva del dolor hasta la remisión completa a las 12 semanas de la operación. A los tres meses, comenzó con gimnasia y, a los seis meses, retornó a la actividad deportiva, sin síntomas.

\section{DISCUSIÓN}

La fractura por estrés del pubis es un problema conocido en la medicina del deporte y tiene una baja frecuencia. ${ }^{12}$ Afecta principalmente a jóvenes y adultos de entre 18 y 55 años que practican una actividad deportiva intensa y tiene una tendencia por el sexo femenino. Está descrita en reclutas militares, corredores de larga distancia, jugadores de fútbol y rugby. También afecta a mujeres $>60$ años, con osteoporosis, luego de un trauma de baja energía o a aquellas que realizan una actividad deportiva de moderada intensidad. ${ }^{13,14}$ Se puede detectar aislada o asociada a una fractura por estrés del sacro, lo que ocasiona una mayor inestabilidad del anillo pélvico. Aretxabala y cols. reportaron una tasa del $78 \%$ de asociación de estas dos fracturas; por lo tanto, el ortopedista debe considerarla en el momento del diagnóstico. ${ }^{15}$ En la revisión bibliográfica, se encontraron 193 casos (Tabla). Dummar y cols. ${ }^{16}$ reportaron un caso de fractura por estrés del pubis en una mujer de 22 años, asociada a neurofibromatosis tipo 1 , lo que interpretamos como un hallazgo dado que no hay demasiada bibliografía que afirme la asociación directa de estas dos enfermedades. Marshall y Gringmuth ${ }^{17}$ publicaron el caso de un joven de 16 años que sufrió una fractura por estrés del pubis luego de una práctica deportiva. Este paciente tenía enfermedad de Crohn, lo que aporta un trastorno nutricional que puede alterar la biología ósea. 
A

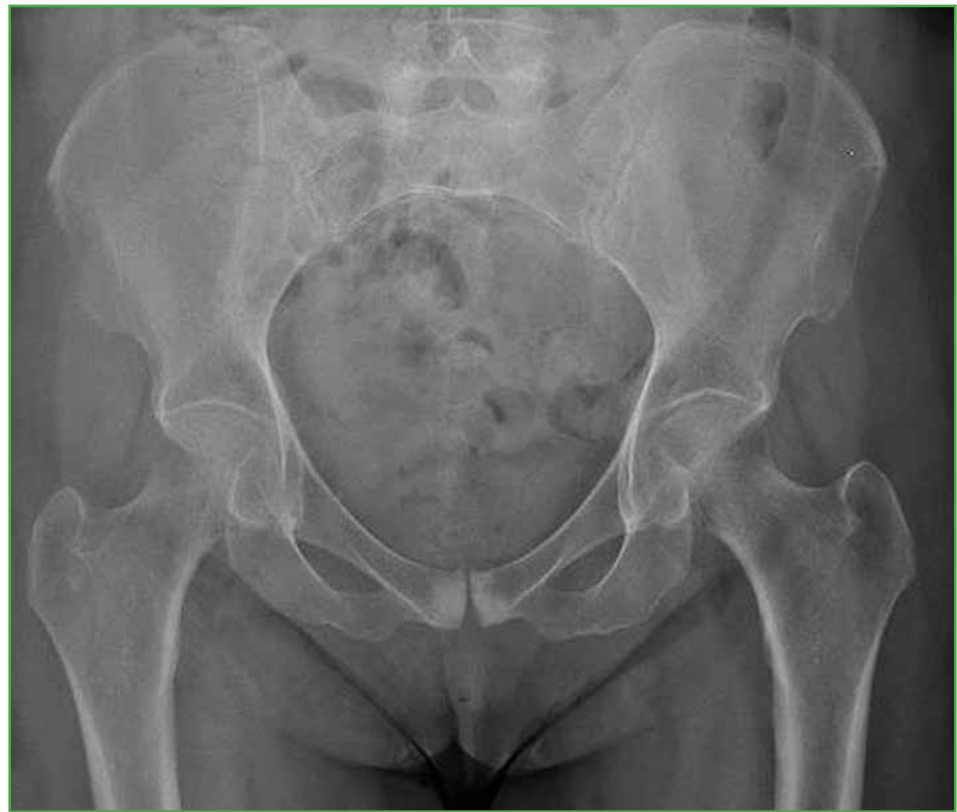

B
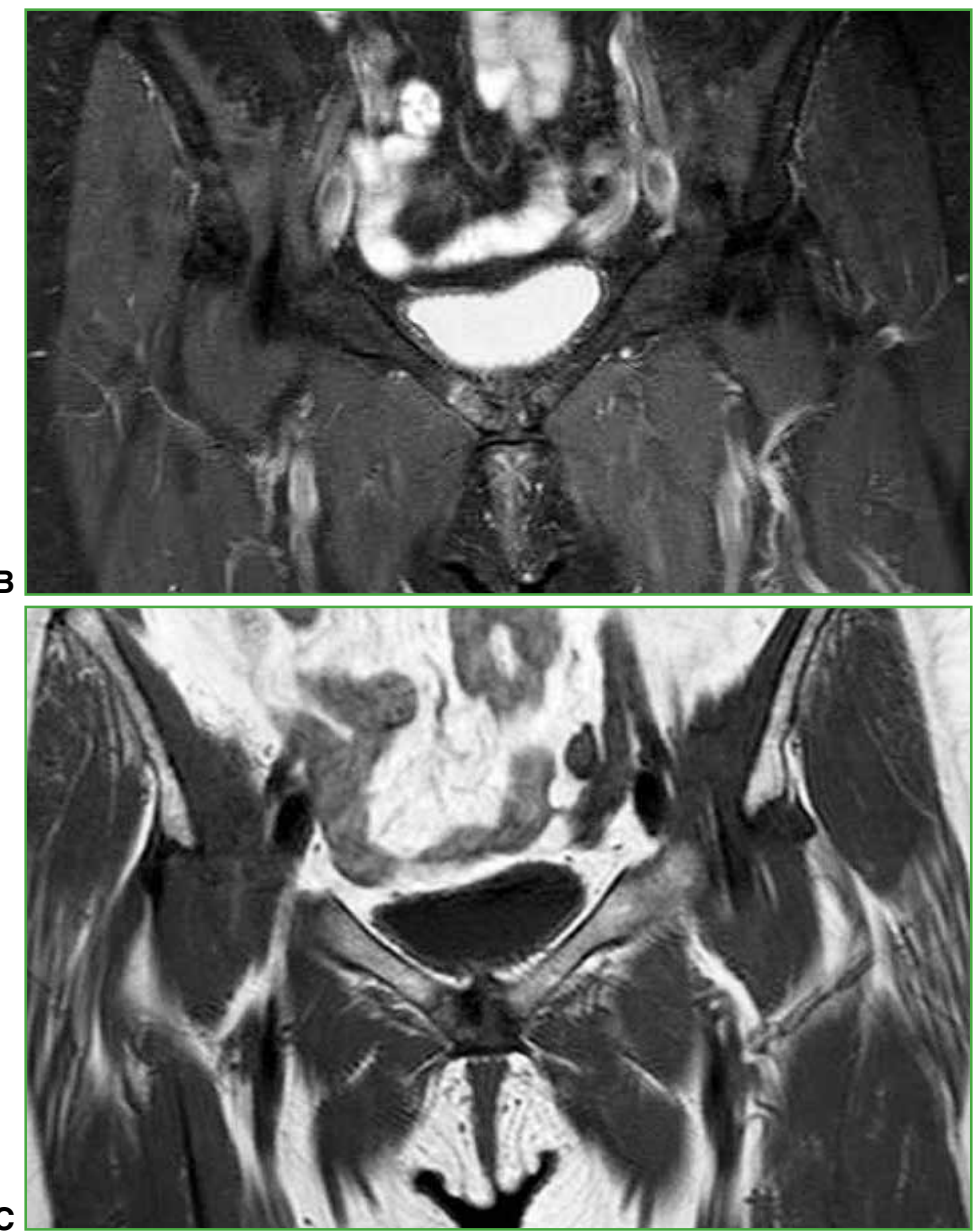

Figura 1. Mujer de 72 años. A. Radiografía anteroposterior de pelvis. Se observa un aumento de la radiopacidad en ambas ramas púbicas. B. Resonancia magnética de pelvis, corte coronal, secuencia STIR. Se visualiza el edema óseo en la rama púbica derecha. C. Resonancia magnética de pelvis, secuencia T1. Se observa el trazo de fractura hipointenso. 
A

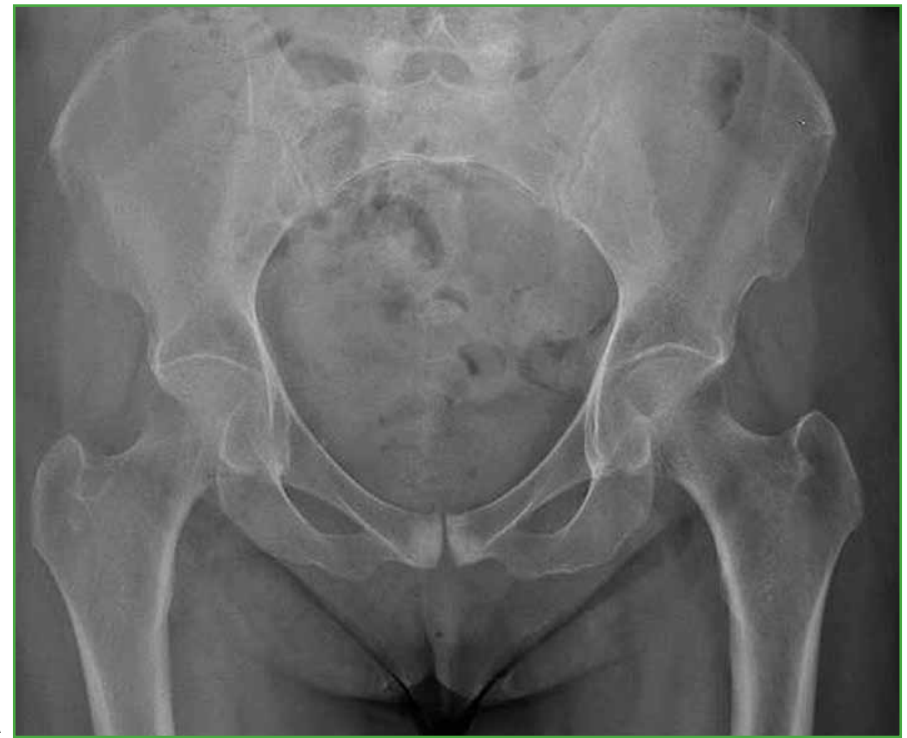

B
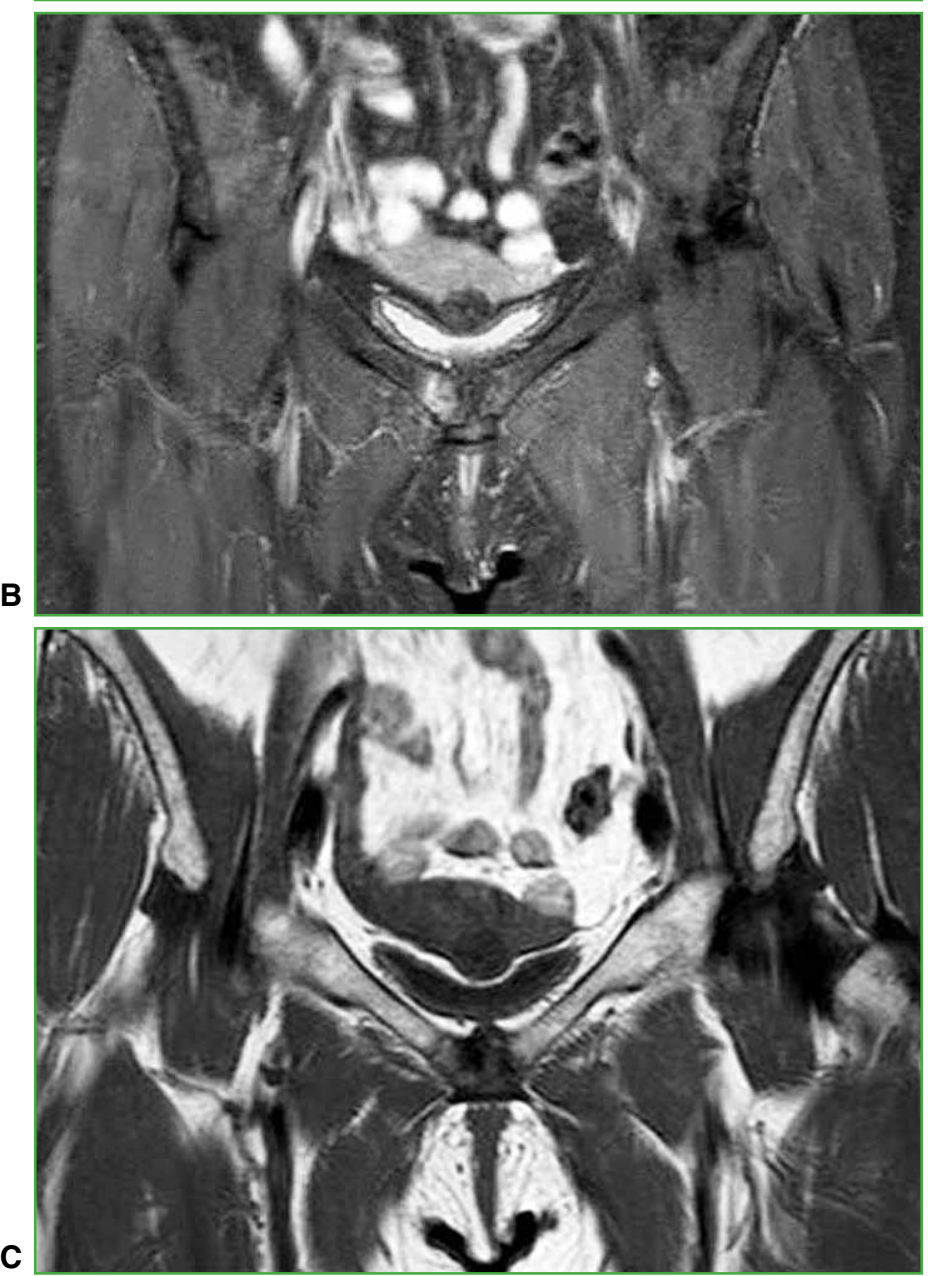

Figura 2. Paciente a los 8 meses. A. Radiografía anteroposterior de pelvis con características idénticas después del tratamiento conservador.

B. Resonancia magnética de pelvis, corte coronal, secuencia STIR. Se puede observar el edema óseo en la rama púbica derecha, signo de ausencia de consolidación de la fractura. C. Resonancia magnética de pelvis, secuencia T1. Se sigue observando el trazo de fractura en la rama púbica derecha. 
A

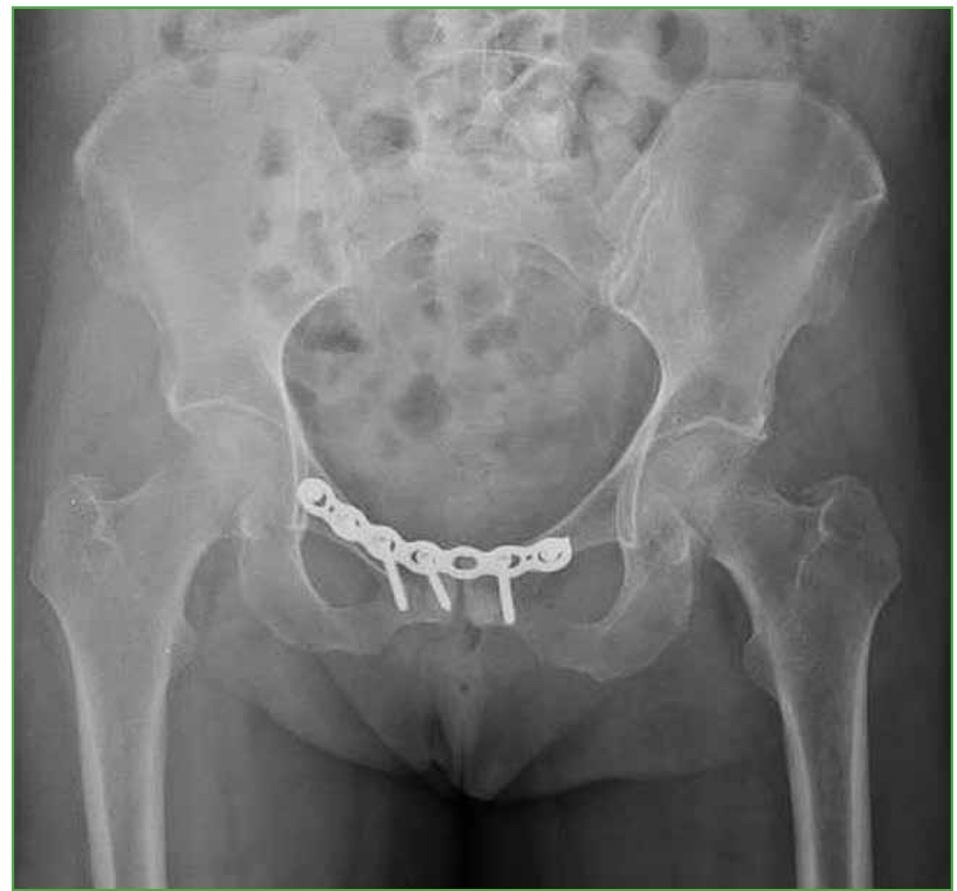

B

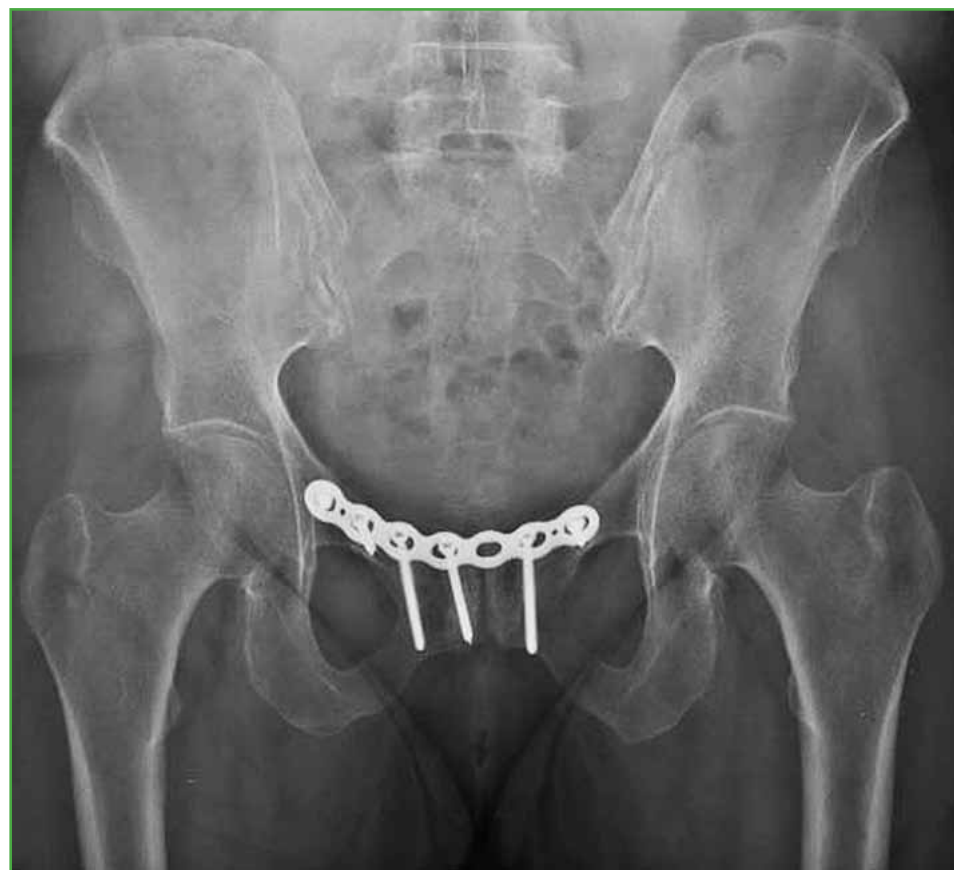

Figura 3. Imágenes clínicas posoperatorias. Se aprecia la placa de osteosíntesis con seis tornillos de fijación. A. Radiografía posoperatoria inmediata.

B. Radiografía a los tres meses. Se visualiza el aumento de la radiopacidad observada al principio del tratamiento. 
Tabla. Características demográficas de los pacientes reportados con fractura por estrés del pubis sin traumatismo directo ni secundario a procedimiento quirúrgico

\begin{tabular}{|c|c|c|c|c|c|}
\hline Autor & Publicación & $\mathbf{n}$ & $\begin{array}{l}\text { Edad } \\
\text { (años) }\end{array}$ & Sexo & Actividad y enfermedades asociadas \\
\hline Selakovich & J Bone Joint Surg Am (1954) & 5 & $20-22$ & M & Reclutas militares \\
\hline Wilson & Radiology (1969) & 1 & NR & M & Reclutas militares \\
\hline Orava & Acta Orthop Scand (1978) & 2 & NR & $\begin{array}{r}\text { M } 1 \\
\text { F } 1\end{array}$ & Corredores \\
\hline Meurman & Br J Radiol (1980) & 49 & $18-29$ & M & Reclutas militares \\
\hline Ozburn & Mil Med (1981) & 70 & 21 & F 67 M 3 & Reclutas militares \\
\hline Tehranzadeh & AJSM (1982) & 1 & 21 & M & Corredor \\
\hline Pavlov & J Bone Joint Surg Am (1982) & 12 & $19-48$ & $\begin{array}{l}\text { F } 2 \\
\text { M } 2\end{array}$ & Maratonistas \\
\hline Noakes & Am J Sports Med (1985) & 5 & $21-54$ & $\begin{array}{l}\text { F } 3 \\
\text { M } 2\end{array}$ & Maratonistas \\
\hline Thorne & Clin Nucl Med (1986) & 1 & 43 & $\mathrm{~F}$ & Maratonista \\
\hline Kim & Clin Nucl Med (1987) & 1 & 39 & M & Nadador \\
\hline Arafat & Ann Rheum Dis (1994) & 1 & 62 & $\mathrm{~F}$ & Artritis reumatoide \\
\hline Hill & J Bone Joint Surg Br (1996) & 11 & NR & $\mathrm{F}$ & Reclutas militares \\
\hline Schapira & Semin Arthritis Rheum (1996) & 7 & NR & $\mathrm{F}$ & Osteoporosis y artritis reumatoide \\
\hline Thienpont & Acta Orthop Belg (2000) & 1 & 23 & M & $\begin{array}{l}\text { Maratonista, hipogonadismo hipogonadotrófico } \\
\text { secundario a anorexia }\end{array}$ \\
\hline Kim & Korean J Intern Med (2000) & 1 & 39 & $\mathrm{~F}$ & Enfermedad mixta del tejido conectivo \\
\hline Korpelainen & $\operatorname{AJSM}(2001)$ & 4 & NR & $\begin{array}{l}\text { F } 2 \\
\text { M } 2\end{array}$ & Maratonistas \\
\hline Iwamoto & J Orthop Sci (2003) & 11 & NR & $\begin{array}{l}\text { F } 7 \\
\text { M } 4\end{array}$ & Fútbol, rugby y corredores \\
\hline Lee & Korean J Radiol (2005) & 3 & $19-22$ & M & Reclutas militares \\
\hline Bertolini y cols. & Rev Bras Ortop (2011) & 1 & 15 & M & Fútbol \\
\hline Eisenstein & BMJ Case Rep (2013) & 2 & $27-31$ & $\mathrm{~F}$ & Reclutas militares \\
\hline El Ghoch & BMJ Case Rep (2014) & 1 & 28 & $\mathrm{~F}$ & Anorexia y actividad deportiva compulsiva \\
\hline Kawano & J Orthop Sci (2016) & 1 & 79 & $\mathrm{~F}$ & Parkinson y osteoporosis \\
\hline $\begin{array}{l}\text { Marshall } \\
\text { y Gringmuth }\end{array}$ & J Can Chiropr Assoc (2019) & 1 & 16 & M & Fútbol + enfermedad de Crohn \\
\hline Dummar y cols. & J Orthop Sports Phys Ther (2019) & 1 & 22 & $\mathrm{~F}$ & Militar con neurofibromatosis \\
\hline Caso actual & & 1 & 72 & $\mathrm{~F}$ & Tenis y golf + osteoporosis \\
\hline
\end{tabular}

$\mathrm{M}=$ masculino, $\mathrm{F}=$ femenino, $\mathrm{NR}=$ no reportado.

La fractura por estrés del pubis es un cuadro de etiología conocida cuyo diagnóstico se basa en los hallazgos clínicos y por imágenes. Si bien debe considerarse como potencial diagnóstico diferencial de un dolor inguinal crónico o recurrente, ${ }^{17}$ el estudio por imágenes es fundamental en el diagnóstico de esta entidad. La radiografía de pelvis con proyección anteroposterior, entrada y salida es el estudio inicial debido a su fácil acceso y bajo costo; sin embargo, hay reportes que informan una tasa alta de resultados falsos negativos. ${ }^{4,18}$ Ante la sospecha de inestabilidad pélvica, las radiografías estáticas son inadecuadas para detectar un movimiento relativo anormal entre ambas hemipelvis. 
En este contexto, se debe evaluar al paciente con radiografías de pelvis dinámicas con carga de peso en un pie y posteriormente en el otro (flamingo view). Garras y cols. sugieren que existe un movimiento fisiológico de $5 \mathrm{~mm}$ en la sínfisis púbica en personas asintomáticas. ${ }^{19}$ La centellografía ósea total tiene una sensibilidad del 74-100\% para la remodelación ósea y muestra alteraciones en las imágenes de tres a cinco días después del comienzo de los síntomas, revela una fractura por estrés días o semanas antes que las radiografías. También es útil para el diagnóstico diferencial cuando se sospechan lesiones metastásicas. ${ }^{718}$ Se recurre a la tomografía computarizada principalmente cuando está contraindicada la resonancia magnética. Las lesiones crónicas pueden ser más evidentes con este estudio que con la resonancia magnética o la centellografía ósea total; sin embargo, deben reservarse solo para indicaciones específicas dado que implica una alta exposición ionizante. La resonancia magnética es el estudio recomendado luego de una radiografía sin alteraciones, es el método más sensible y específico para el diagnóstico de este cuadro, además, ofrece información sobre la gravedad y el pronóstico de la lesión. ${ }^{4,7}$

Con el fin de realizar un tratamiento adecuado de la fractura por estrés del pubis, es esencial identificar los factores de riesgo para el desarrollo de este cuadro. La terapia debería basarse en la prevención de nuevos episodios y la recuperación de la fractura. ${ }^{4,20} \mathrm{El}$ cambio de actividades, la corrección de malos hábitos deportivos, modificar el equipamiento y la superficie de práctica deportiva, optimizar los hábitos nutricionales, reconocer alteraciones hormonales y anatómicas, la fuerza muscular y la aptitud cardiovascular podrían ser de gran importancia para prevenir nuevas lesiones. El tratamiento tradicional incluye el manejo del dolor, la rehabilitación física y reducir, al mínimo, la sobrecarga del sitio afectado sin evitar las actividades de la vida diaria, inclusive la descarga en ambos miembros desde el inicio., ${ }^{4,20}$ A pesar del tratamiento conservador temprano con las medidas antes descritas, el alivio del dolor y el retorno a la actividad previa a la lesión pueden demorar de 4 a 12 semanas. ${ }^{7,20}$

La fractura por estrés del pubis representa un desafío cuando ocurre en mujeres añosas con osteoporosis que mantienen una práctica deportiva periódica, ya que la consolidación sobre un hueso de mala calidad se torna un problema. Por lo tanto, las medidas conservadoras podrían ser insuficientes. Si bien el tratamiento recomendado para una fractura por fragilidad de la pelvis tipo Ia, según la clasificación propuesta por Rommens y Hofmann, ${ }^{21}$ es el conservador, las complicaciones mencionadas en personas mayores varían desde una infección del tracto urinario y de las vías respiratorias bajas hasta un tromboembolismo pulmonar. Además, la estancia hospitalaria y los servicios de atención domiciliaria necesarios en esta población luego de sufrir esta fractura implican un alto costo económico para el sistema de salud. ${ }^{22,23}$ Por lo tanto, creemos que la indicación del tratamiento depende de las condiciones generales y las enfermedades asociadas del paciente.

A pesar de que las complicaciones asociadas al tratamiento médico son menores comparadas con las del tratamiento quirúrgico, Höch y cols. mostraron que la supervivencia a mediano plazo, luego de dos años, fue mayor en los pacientes con fracturas estabilizadas quirúrgicamente..$^{24}$

En nuestro caso, la paciente posmenopáusica con un índice de masa corporal de 21, osteoporosis diagnosticada por densitometría ósea y tratada junto con el Servicio de Reumatología, y el antecedente de tabaquismo constituyeron los factores de riesgo más relevantes para decidir entre los especialistas del equipo médico y la paciente un manejo quirúrgico teniendo en cuenta que, luego de seis meses, el tratamiento primario no resultó adecuado y la paciente deseaba recuperarse rápidamente para continuar con sus actividades diarias y deportivas. El seguimiento radiográfico mostró una buena evolución y el dolor en la región inguinal desapareció a los tres meses de la cirugía; la paciente reanudó su actividad deportiva a los seis meses.

\section{CONCLUSIONES}

La fractura por estrés del pubis es una entidad conocida y poco frecuente asociada a la fragilidad ósea y a una actividad deportiva intensa. El diagnóstico es clínico y por imágenes. El tratamiento depende del tipo de fractura, las condiciones generales y las enfermedades asociadas. La indicación del tratamiento no solo debe limitarse al grado de inestabilidad de la fractura, sino que también debe tener en cuenta el contexto médico y social del paciente.

Conflicto de intereses: Los autores no declaran conflictos de intereses. 


\section{BIBLIOGRAFÍA}

1. Hosey RG, Fernandez MM, Johnson DL. Evaluation and management of stress fractures of the pelvis and sacrum. Orthopedics 2008;31:383-5. https://doi.org/10.3928/01477447-20080401-14

2. Snyder RA, Koester MC, Dunn WR. Epidemiology of stress fractures. Clin Sports Med 2006;25(1):37-52. https://doi.org/10.1016/j.csm.2005.08.005

3. Miller C, Major N, Toth A. Pelvic stress injuries in the athlete. Sports Med 2003;33:1003-12. https://doi.org/10.2165/00007256-200333130-00005

4. Carmont RC, Mei-Dan O, Bennell KL. Stress fracture management: current classification and new healing modalities. Oper Tech Sports Med 2009;17:81-9. https://doi.org/10.1053/j.otsm.2009.05.004

5. Quinteros C. Fractura por estrés de rama iliopubiana - Reporte de caso. Revista de la Facultad de Ciencias Médicas de Córdoba 2018;135-6. https://doi.org/10.31053/1853.0605.v0.n0.21473

6. Bennell KL, Malcolm SA, Thomas SA, Wark JD, Brukner PD. The incidence and distribution of stress fractures in competitive track and field athletes. A twelve-month prospective study. Am J Sports Med 1996;24(2):211-7. https://doi.org/10.1177/036354659602400217

7. Costa Astur D, Zanatta F, Gonçalves Arliani G, Moraes ER, de Castro Pochini A, Ejnisman B. Stress fractures: definition, diagnosis and treatment. Rev Bras Ortop 2016;51(1):3-10. https://doi.org/10.1016/j.rboe.2015.12.008

8. Dauty M, Collon S. Incidence of injuries in French professional soccer players. Int J Sports Med 2011;32(12):965-9. https://doi.org/10.1055/s-0031-1283188

9. Pepper M, Akuthota V, McCarty EC. The pathophysiology of stress fractures. Clin Sports Med 2006;25(1):1-6. https://doi.org/10.1016/j.csm.2005.08.010

10. Joy EA, Campbell D. Stress fractures in the female athlete. Curr Sports Med Rep 2005;4(6):323-8. https://doi.org/10.1097/01.csmr.0000306294.72578.a8

11. Henning PT. The running athlete: stress fractures, osteitis pubis, and snapping hips. Sports Health 2014;6(2):122-7. https://doi.org/10.1177/1941738114523381

12. Bertolini FM, Vieira RB, Araujo de Oliveira LH, Lasmar RP, Taviano de Oliveira J. Pubis stress fracture in a 15 year old soccer player. Rev Bras Ortop 2011;46(4):464-7. https://doi.org/10.1016/S2255-4971(15)30263-9

13. Tacettin Ayanaoğlu, O Şahap Atik, Nil Tokgöz, Murat Uçar. Sacral and pubic insufficiency fractures due to bisphosphonate treatment. Eklem Hastalik Cerrahisi 2015;26(2):120-4. https://doi.org/10.5606/ehc.2015.23

14. Ragnarsson B, Jacobsson B. Epidemiology of pelvic fractures in a Swedish county. Acta Orthop Scand 1992;63:297-300. https://doi.org/10.3109/17453679209154786

15. Aretxabala I, Fraiz E, Pérez-Ruiz F, Ríos G, Calabozo M, Alonso-Ruiz A. Sacral insufficiency fractures. High association with pubic rami fractures. Clin Rheumatol 2000;19:399-401. https://doi.org/10.1007/p100011178

16. Dummar AR, Tragord B, Doherty BT. Plexiform neurofibromatosis in a woman with a pubic ramus stress fracture. $J$ Orthop Sports Phys Ther 2019;49(1):37. https://doi.org/10.2519/jospt.2019.7495

17. Marshall C, Gringmuth R. Pubic stress fracture presenting as a strain of adductor longus in a 16-year-old elite soccer player with Crohn's disease: a case report. J Can Chiropr Assoc 2019;63(3):19-204. PMID: 31988541

18. Daffner RH, Pavlov H. Stress fractures: current concepts. Am J Roentgenol 1992;159(2):245-52. https://doi.org/10.2214/ajr.159.2.1632335

19. Garras DN, Carothers JT, Olson SA. Single-leg-stance (Flamingo) radiographs to assess pelvic instability: how much motion is normal? J Bone Joint Surg Am 2008;90(10):2114-8. https://doi.org/10.2106/JBJS.G.00277

20. Raasch WG, Hergan DJ. Treatment of stress fractures: the fundamentals. Clin Sports Med 2006;25(1):29-36. https://doi.org/10.1016/j.csm.2005.08.013

21. Rommens PM, Hofmann A. Comprehensive classification of fragility fractures of the pelvic ring: recommendations for surgical treatment. Injury 2013;44(12):1733-44. https://doi.org/10.1016/j.injury.2013.06.023

22. Taillandier J, Langue F, Alemanni M, Taillandier-Heriche E. Mortality and functional outcomes of pelvic insufficiency fractures in older patients. Joint Bone Spine 2003;70:287-9. https://doi.org/10.1016/s1297-319x(03)00015-0

23. Spencer JL, Lalanadham T. The mortality of patients with minor fractures of the pelvis. Injury 1985;16:321-3. https://doi.org/10.1016/0020-1383(85)90136-6

24. Höch A, Özkurtul O, Pieroh P, Josten C, Böhme J. Outcome and 2-year survival rate in elderly patients with lateral compression fractures of the pelvis. Geriatr Orthop Surg Rehabil 2017;8:3-9.

https://doi.org/10.1177/2151458516681142 